TRANSACTIONS OF THE

AMERICAN MATHEMATICAL SOCIETY

Volume 147, February 1970

\title{
SLICES IN TRANSFORMATION GROUPS
}

\author{
BY
}

\author{
LARRY M. KING
}

1. Introduction. If $(X, T, \pi)$ is a transformation group with $T$ a compact Lie group and $X$ a completely regular space, then it is well known (see Palais [4]) that there exists a slice at every point $x \in X$. Existence of slices facilitates the study of transformation groups since, for example, it enables the reduction of global questions about transformation groups to local ones.

In topological dynamics, transformation groups $(X, T, \pi)$ are studied where $T$ is not compact. In general circumstances like this, it is no longer true that a slice exists at each point of $X$. Generalizing the case when $T$ is compact, Palais [4] showed that if $(X, T, \pi)$ is a Cartan transformation group then slices still exist at each point. Finally Chu [1] showed that if $(X, T, \pi)$ has certain dynamical properties, then there exists some sort of slice through each point.

We investigate what can be said further about the existence of slices in a transformation group $(X, T, \pi)$ where $T$ is not compact.

An example of a transformation group is given which does not fall into the categories studied by Palais or Chu but which nonetheless has a slice at each point. The next example has the same group and phase space with a somewhat different action and we show that there are no slices at any point. Hence, unlike the compact case, the question of existence of slices depends on more than the nature of the group and of the phase space.

Let $(X, T, \pi)$ be a transformation group and $H$ a closed subgroup of $T$. Then $(X, H, \pi)$ is a transformation group. If $S$ is a slice at $x \in X$ in $(X, H, \pi)$, necessary and sufficient conditions are given for $S$ to be a slice at $x$ in $(X, T, \pi)$. Application of this result gives existence of slices in certain non-Cartan tgs. The author especially wishes to thank the referee for suggesting Example 3, an example which illustrates one such application.

We further exhibit necessary and sufficient conditions for a slice $S$ at $x$ in $(X, H, \pi)$ to give a slice $S^{\prime}$ at $x$ in the transformation group $(X, T, \pi)$ with $S^{\prime}$ not necessarily equal to $S$.

If $S_{1}$ and $S_{2}$ are two slices in $(X, T, \pi)$ then in the general case their union is not again a slice, even if $S_{1}$ and $S_{2}$ are disjoint. The notion of a closed slice, somewhat more restrictive than a slice, is introduced and a method of combining closed slices, in certain transformation groups at least, is developed. In this way, if $T=V K$, semidirect product of a vector group and a compact normal subgroup, then the

Received by the editors April 30, 1969. 
existence of a slice at each point of $(X, T, \pi)$ implies the existence of a global slice.

The author wishes to thank Professor Hsin Chu for all his encouragement and help and useful suggestions.

2. Definitions and examples. A transformation group is a triple $(X, T, \pi)$ where $X$ is a Hausdorff space, $T$ a topological group and $\pi: X \times T \rightarrow X$ a continuous map such that $(x, 1) \pi=x$ for $x \in X$ and 1 the identity of $T$ and $((x, t) \pi, s) \pi=(x, t s) \pi$ for $t, s \in T$ and $x \in X$. To facilitate notation, $x t$ is written for $(x, t) \pi$. The isotropy subgroup at $x \in X$, denoted $T_{x}$ is equal to $\{t \in T \mid x t=x\} . x T=\{x t \mid t \in T\}$ is the orbit of $x$. The set consisting of all orbits is denoted by $X / T$ and given the usual quotient topology. For $L$ a closed subgroup of $T, T / L=\{L t \mid t \in T\}$ denotes the space of right cosets. If $S \subset X$ and $F \subset T$ then $S F=\{s f \mid s \in S, f \in F\}$. If $(X, T, \pi)$ and $(Y, T, \rho)$ are two transformation groups then $f: X \rightarrow Y$ is said to be equivariant if for $t \in T, x \in X, f(x t)=[f(x)] t$.

Henceforth the term transformation group will be abbreviated tg and the $\mathrm{tg}$ $(X, T, \pi)$ will be denoted by $(X, T)$ provided that there is no possibility of ambiguity.

In what follows, let $(X, T)$ be a $\operatorname{tg}$ and $L$ a closed subgroup of $T$. If $H$ is a subset of $T$ and $A$ and $B$ are subsets of $X$ then $H(A, B)=\{t \in H \mid A t \cap B \neq \varnothing\}$.

$S \subset X$ is an $L$-slice in the $\operatorname{tg}(X, T)$ if

(i) $S L=S$.

(ii) $T(S, S) \subset L$.

(iii) If $U$ is open in $T$, then $S U$ is open in $X$.

If $x \in X, L=T_{x}$ and $x \in S$ then $S$ is said to be a slice at $x$.

$S$ is a global $L$-slice if $S$ is an $L$-slice and in addition $S T=X$. The following remark indicates that this definition of slice is equivalent to that used by Palais [4].

RemarK. $S$ is an $L$-slice in the $\operatorname{tg}(X, T)$ if and only if there exists a continuous equivariant map $f: S T \rightarrow T / L$ such that $f^{-1}(L)=S$ and $S T$ is open in $X$.

It is known that a slice exists at each point $x \in X$ of a $\operatorname{tg}(X, T)$ if $T$ is a compact Lie group. Palais generalized this result to a larger class of tgs, namely to Cartan tgs.

A $\operatorname{tg}(X, T)$ is Cartan if for $x \in X$ there is a neighborhood $U$ of $x$ such that $T(U, U)$ is contained in a compact set.

Theorem (PAlAis). Let $(X, T)$ be a tg with $T$ a Lie group and $X$ a completely regular space. Then $(X, T)$ is Cartan if and only if for $x \in X, T_{x}$ is compact and there is a slice at $x$.

In order to prove this theorem it was necessary to introduce the notion of a proper tg which is a more restrictive property than that of being Cartan.

A $\operatorname{tg}(X, T)$ is proper if for $x \in X$ there exists a neighborhood $U$ of $x$ such that for any $y \in X$ there is a neighborhood $V$ of $y$ such that $T(U, V)$ is contained in a compact set where $U$ depends only on $x$ and not on the choice of $y$.

The following examples give some indication of the complexity of the problem of determining whether slices exist in tgs. 
Example 1. Let $X$ be the Euclidean plane and $H=K$ be the additive group of real numbers. Let $T=H \times K$, direct product. We make $(X, T)$ a tg by defining the action of $T$ on $X$ to be vertical translation as follows: for $(x, y) \in X,(h, k) \in T$ define $(x, y)(h, k)=(x, y+h-k)$. It is easy to see that $(X, T)$ is not a Cartan $\operatorname{tg}$ yet there exists a slice at every point of $X$.

Example 2. Let $X$ and $T$ be as in Example 1. Define the action of $T$ on $X$ as follows: for $(h, k) \in T,(x, y) \in X$ define $(x, y)(h, k)=(x, y-h+k x)$. It turns out that in this tg there is no slice at any point. In fact, assuming a slice $S$ exists at some point it can be shown that $S$ consists of a single point which clearly gives rise to a contradiction. Observe further that the tgs $(X, H)$ and $(X, K)$ each have a slice at every point of $X$.

3. Slices in non-Cartan transformation groups. Let $(X, T, \pi)$ be a tg and $H$ a closed subgroup of $T$. Then $\left(X, H, \pi_{H}\right)$ is also a tg where $\pi_{H}$ is the restriction of $\pi$ to $X \times H$. $\left(X, H, \pi_{H}\right)$, written $(X, H)$ when no ambiguity results, is called a subtransformation group, abbreviated stg, of $(X, T)$.

It is possible for a stg $(X, H)$ of $(X, T)$ to be Cartan while $(X, T)$ is not. Examples 1 and 2 both give instances of this possibility. Hence it is conceivable that a stg $(X, H)$ of $(X, T)$ may have a slice at each point while $(X, T)$ does not. Example 2 is such an example.

In this section we provide necessary and sufficient conditions for the existence of a slice in a stg to imply the existence of a slice in the tg.

THEOREM 1. Let $(X, T)$ be $a$ tg and $H$ a closed subgroup of $T$. Let $x \in X$ and $S$ be a slice at $x$ in the stg $(X, H)$. Suppose in addition that $S T$ is connected. Then the following statements are equivalent:

(a) (i) $S$ is a slice at $x$ in $(X, T)$.

(ii) $T_{x} H$ is closed in $T$.

(b) (i) $S T=S H$.

(ii) $T(S, S) \subset T_{x}$.

(c) (i) $S T_{x}=S$.

(ii) $T=T_{x} H$.

Proof. In order to show that (a) implies (b) consider the equivariant map defining the slice $S$; i.e., $f: S T \rightarrow T / T_{x}$. Since $T_{x} H$ is closed in $T$, it follows that if $\rho: T \rightarrow T / T_{x}$ is the nat 'ural projection then $\rho(H)$ is closed and $f^{-1}[\rho(H)]=S H$ is closed in $S T$. On the other hand since $S$ is a slice in $(X, T), S H$ is open in $X$ and a fortiori open in $S T$. Since $S T$ is connected $S T=S H$. Finally (a) (i) immediately gives (b) (ii).

For the converse, one first observes by using a standard net argument (see for example the proof of Corollary 1 below) that $S H$ closed in $S T$ implies $T_{x} H$ is closed in $T$. To show that $S$ is a slice at $x$ in $(X, T)$, a continuous equivariant map $f: S T \rightarrow T / T_{x}$ is constructed by composing the following maps.

Let $i: S T \rightarrow S H$ be the identity map. That is, for $t \in T, s \in S, i(s t)=s_{1} h$ where $h \in H$ and $s_{1} \in S$ so that $s t=s_{1} h$. Let $f_{1}: S H \rightarrow H / H_{x}$ be the continuous equivariant 
map defining the slice at $x$ in $(X, H)$. Finally let $t: H / H_{x} \rightarrow T / T_{x}$ be defined by $t\left(H_{x} h\right)=T_{x} h$.

If $f=t \circ f_{1} \circ i$ then $f$ has all the desired properties.

To show that (b) implies (c) we observe first that $S T_{x}=S$ since by (a) $S$ is a slice at $x$ in $(X, T)$ and so invariant under $T_{x}$. Now let $t \in T$. Select $s_{1}, s_{2} \in S$ and $h \in H$ such that $s_{1} t=s_{2} h$. By (b)(ii) it follows that $t h^{-1} \in T_{x}$ and so $t \in T_{x} H$. Hence $T=T_{x} H$ and so the proof of this implication is complete.

Finally (b)(i) follows easily from (c)(i) and (ii) since $S T=S T_{x} H=S H$. For (b)(ii) let $t \in T(S, S)$. Then $s t \in S$ for some $s \in S$. (c)(ii) implies that $t=g h$ for some $g \in T_{x}$ and $h \in H$. This together with the fact that we are assuming that $S$ is a slice at $x$ in $(X, H)$ gives that $t \in T_{x}$.

Corollary 1. Let $(X, T)$ be a tg, $H$ a closed subgroup of $T$ and $(X, H)$ a Cartan stg of $(X, T)$. Let $S$ be a slice at $x$ in $(X, H)$. If $S T$ is connected then $S$ is a slice at $x$ in $(X, T)$ if and only if $S T=S H$ and $T(S, S) \subset T_{x}$.

Proof. It is enough to show that $T_{x} H$ is closed in $T$ and then apply Theorem 1. In fact we observe that since $(X, H)$ is a Cartan $\operatorname{tg}, x H$, for $x \in X$ is closed. Let $\left\{t_{\alpha} h_{\alpha}\right\}$ be a net in $T_{x} H$ with $\left\{h_{\alpha}\right\} \subset H,\left\{t_{\alpha}\right\} \subset T_{x}$ and $t_{\alpha} h_{\alpha} \rightarrow t$ for some $t \in T$. Hence $x t_{\alpha} h_{\alpha} \rightarrow x t$ and so $x h_{\alpha} \rightarrow x t$. Since $x H$ is closed $x t=x h$ for some $h \in H$ and therefore $t \in T_{x} H$.

COROLlary 2. Let $(X, T)$ be a tg with $X$ a completely regular space, $T$ a Lie group and $H$ a closed subgroup of $T$. Let $(X, H)$ be a Cartan stg of $(X, T)$ and let there exist a group homomorphism $f: T \rightarrow H$ such that

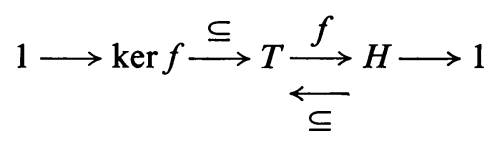

is a split exact sequence with $\operatorname{ker} f \subset \bigcap_{x \in X} T_{x}$. Then for $x \in X$ there is a slice at $x$ in $(X, T)$.

Proof. Let $x \in X$. Palais' theorem gives the existence of a slice $S$ at $x$ in the $\operatorname{stg}(X, H)$. It is clear that $T(S, S) \subset T_{x}$ and that $S T=S H$. Now apply Corollary 1.

Corollary 2 takes care of cases exhibited by Example 1 . A $\operatorname{tg}(X, T)$ satisfying the hypotheses of this corollary is not in general a Cartan tg. Hence we have the existence of a slice at every point in a $\operatorname{tg}(X, T)$ which is more general than a Cartan tg. If $(X, T)$ is itself Cartan, let $H=T$ and the hypotheses of the Corollary are satisfied.

Perhaps a more important situation covered by Theorem 1 is the following. Suppose $(M, T)$ is a $\operatorname{tg}$ with $M$ a $C^{\infty}$ differentiable manifold and $T$ a Lie group acting smoothly on $M$. It is well known that the action can be lifted to a smooth action on the tangent bundle $T(M)$ of $M$. If $H$ is a compact subgroup of $T$ then slices exist in $(T(M), H)$. Theorem 1 may apply in such a case to give slices in the $\operatorname{tg}(T(M), T)$. 
ExAmPle 3. Let $T=G L(n+1, \mathbf{R}) /$ center, the projective linear group (where the center is the collection of scalar matrices). Let $\mathbf{R} P^{n}$ be projective $n$-space represented as the collection of lines through the origin in $\mathbf{R}^{n+1} . T$ acts smoothly on $\mathbf{R} P^{n}$ and so acts on its tangent bundle $X=T\left(\mathbf{R} P^{n}\right)$.

If $x$ is any point of the zero section of $X$, say over the base point $p \in \mathbf{R} P^{n}$, let $S$ be the fiber of $X$ at $p$. If $H=S O(n+1) \subset T$ then $S$ is a global slice at $x$ in $(X, H)$ and since $T(S, S) \subset T_{x}, S$ is a global slice at $x$ in $(X, T)$.

Since, for example $T_{x}$ is isomorphic to $G L(n) /$ center and so not compact $(X, T)$ is not Cartan. Moreover, Corollary 2 does not cover this situation since no homomorphism $f: T \rightarrow H$ as called for in the corollary exists because the only nontrivial normal subgroups of $T$ contain $H$.

If $(X, T)$ is a tg, $H$ a closed subgroup of $T$ and $(X, H)$ a Cartan stg of $(X, T)$, then $T_{x} H$ is closed in $T$. If the hypothesis that $(X, H)$ is Cartan is removed, then $T_{x} H$ is no longer necessarily closed in $T$. In what follows, the hypothesis that $T_{x} H$ be closed is removed and replaced by that of $H$ being a normal subgroup of $T$.

THEOREM 2. Let $(X, T)$ be a tg where $T$ is connected and let $H$ be a closed normal subgroup of T. Let $x \in X$ and $S$ be a connected slice at $x$ in the stg $(X, H)$. Then $S$ is a slice at $x$ in $(X, T)$ if and only if $S T=S H$ and $T(S, S) \subset T_{x}$.

Proof. That $S$ is a slice at $x$ in $(X, T)$ follows directly from Theorem 1 . The proof of the converse is broken up into two cases.

Case 1. If $T_{x} H$ is closed in $T$ then again Theorem 1 applies.

Case 2. $T_{x} H$ is not closed. Let $S$ be the slice at $x$ in tgs $(X, T)$ and $(X, H)$. Since $H$ is a normal subgroup of $T, T_{x} H$ is itself a subgroup. Hence $T_{x} H$ is not open in $T$ and so $x H$ is not open in $x T$. Therefore $S$ cannot be a slice at $x$ in $(X, T)$ and we conclude that Case 2 is impossible.

Up to this point, only the question of when a slice $S$ in $(X, H)$ at a point $x \in X$ is a slice at $x$ in $(X, T)$ has been considered. We now investigate when the existence of a slice in $(X, H)$ implies the existence of an $L$-slice $S^{\prime}$ in $(X, T)$ where $S$ does not equal $S^{\prime}$ in general and $L \neq T_{x}$.

THEOREM 3. Let $(X, T)$ be a tg and $(X, H)$ a stg, for $H$ a closed subgroup of $T$. Let $K$ be a closed subgroup of $T$. If $S$ is an L-slice containing $x$ in $(X, H), L$ a closed subgroup of $H$, then let $S^{\prime}=S K$. If $T(S, S) \subset K$ then $S^{\prime}$ is a $K$-slice in the $\operatorname{tg}(X, T)$.

Proof. If we can show that $U$ open in $T$ implies that $S^{\prime} U$ is open in $X$, then it is clear that $S^{\prime}$ is indeed a slice in $(X, T)$. Suppose therefore that $U$ is open in $T$. For $t \in T$, define $U^{t}=U \cap H t$. $U^{t} t^{-1}$ is open in $H$ and since $U=\bigcup_{t \in T} U^{t}, S U^{t} t^{-1}$ is open in $X$ and so $S U$ is open. If $U$ is open in $T, K U$ is open and so $S^{\prime} U$ is open in $X$.

Corollary. Let $(X, T)$ be a tg and $(X, H)$ a stg for $H$ a closed subgroup of $T$. Let $S$ be a slice at $x$ in $(X, H)$. If $T(S, S)=K$ is a closed subgroup of $T$ then $S^{\prime}=S K$ is a $K$-slice in $(X, T)$. 
We conclude this section with an example that shows that if the hypothesis of the corollary holds then $K=T(S, S)$ may not be closed and may not be a subgroup of $T$.

Example 4. Let $T=\mathbf{R}$ and $X=\mathbf{R}$. $T$ acts on $X$ by translation. That is, for $t \in T$, $x \in X, x t=x+t$. Let $H=Z$ be the integer group and let $x=0$. It is easy to see that $S=\left\{x \in X|| x \mid<\frac{1}{2}\right\}$ is a slice at 0 in the $\operatorname{stg}(X, Z)$.

$$
K=\mathbf{R}(S, S)=\{r \in \mathbf{R} \mid r+s \in S \text {, for some } s \in S\}=\{r \in \mathbf{R}|| r \mid<1\} .
$$

4. Closed slices. Consider the $\operatorname{tg}(X, T)$ as in Example 4. For each $x \in X$, $T_{x}=\{0\}$. Let $S_{1}=\left\{x \in X|| x \mid<\frac{1}{4}\right\}$ and $S_{2}=\left\{x \in X|| x-1 \mid<\frac{1}{4}\right\}$. Since $S_{1}$ and $S_{2}$ are open intervals each of length one-half it follows that $S_{1}$ and $S_{2}$ are $\{0\}$-slices in the $\operatorname{tg}(X, T) . S_{1} \cap S_{2}=\varnothing$ yet $S_{1} \cup S_{2}$ is not a $\{0\}$-slice since $T\left(S_{1} \cup S_{2}, S_{1} \cup S_{2}\right)$ $\neq\{0\}$. In fact, the only subgroup containing all of $T\left(S_{1} \cup S_{2}, S_{1} \cup S_{2}\right)$ is $T$ itself.

So in general, the union of two slices is not again a slice. It would be desirable to have a method of combining slices in some way to obtain larger slices so that, for example, a slice at each point of a tg would imply the existence of a global slice.

The notion of a closed slice in a tg is introduced in this section in order to develop, at least in certain special cases, a process for combining slices to obtain larger ones.

$S$ is a closed $L$-slice in the $\operatorname{tg}(X, T)$, for $L$ a closed subgroup of $T$ if

(i) $S L=S$.

(ii) $T(S, S) \subset L$.

(iii) If $U$ is open in $T$, then $S U$ is open in $S T$.

(iv) $S T$ is closed.

(v) There exists an $S^{1} \subset S$ such that $S^{1} T$ is open.

$S$ is a closed slice at $x \in X$ if $S$ is a closed $L$-slice with $L=T_{x}$ and $x \in S^{1}$. The notions of global closed slice and global slice coincide.

REMARK. Let $S$ be a closed $L$-slice in $(X, T)$, for $L$ a closed subgroup of $T$ with $S^{1} \subset S$ as required by the definition. Then $S^{1} L=S_{1} \subset S$ is an $L$-slice. Conversely if $S$ is an $L$-slice and $x \in S$ then there exists a closed $L$-slice $S_{2} \subset S, x \in S_{2}$ if the space $X / T$ of orbits is regular.

This section was prefaced with the remark that the union of slices is not in general a slice. In what sense then can we speak of larger slices?

If $S_{1}$ and $S_{2}$ are two slices and if $S$ is a slice such that $S T=S_{1} T \cup S_{2} T$ then $S$ is said to be larger than $S_{1}$ and $S_{2}$. This condition amply compensates for the larger slice $S$ not being the union of $S_{1}$ and $S_{2}$.

The first theorem gives a method for combining closed slices. The following is a preliminary lemma.

LEMma. Let $X$ be a normal space and $(X, T)$ a $\mathrm{tg}$ with $T$ a vector group. Let $S_{1}$ and $S_{2}$ be two closed 1-slices where 1 is the identity of $T$. Then there exists a closed 1 -slice $S \supset S_{1}$ that is larger than $S_{1}$ and $S_{2}$. 
Proof. Since $S_{i}, i=1,2$ are closed 1-slices, there exist continuous equivariant maps $f_{i}: S_{i} T \rightarrow T$ with $f_{i}^{-1}(1)=S_{i}$. Let $g: S_{1} T \cap S_{2} \rightarrow T$ be the continuous map defined by $g\left(s_{1} t\right)=t^{-1}$ for $t \in T, s_{1} \in S_{1}$. Since $S_{1} T \cap S_{2}$ is closed, $X$ is a normal space and $T$ is a vector group, Tietze's Extension Theorem extends $g$ in a continuous fashion to all of $S_{2}$. For ease of notation, the extension will be called $g$ also. Define $G: S_{2} \rightarrow X$ by $G(s)=s g(s)$ for $s \in S_{2}$. $G$ is continuous since $g$ is. Let $S=S_{1} \cup G\left(S_{2}\right)$. By exhibiting a continuous equivariant map $f: S T \rightarrow T$ with $f^{-1}(1)=S$ one easily shows that $S$ is the desired larger closed 1-slice.

REMARK. In the above lemma, $S$ is not equal to the union of $S_{1}$ and $S_{2}$ but rather to the union of $S_{1}$ and a homeomorphic image of $S_{2}$ contained in $S_{2} T$.

Theorem 4. Let $X$ be a normal space and $(X, T)$ a tg where $T=K V$, semidirect product of $V$, a vector group and $K$ a compact normal subgroup of $T$. Suppose $S_{1}$ is a closed $L_{1}$-slice and $S_{2}$ is a closed $L_{2}$-slice where $L_{1}$ and $L_{2}$ are closed subgroups of $K$. Then there is a closed $K$-slice $S \supset S_{1}$ such that $S$ is larger than $S_{1}$ and $S_{2}$.

Proof. Let $\bar{T}=T / K$ and $\bar{X}=X / K$, the space of $K$-orbits of $X$. It is known that $\bar{T}$ is a vector group and that $\bar{X}$ is a normal space. If $p: X \rightarrow \bar{X}$ defined by $p(x)=\bar{x}=x K$ for $x \in X$ is the natural projection then $p$ is open, continuous and closed. For $\bar{x} \in \bar{X}, \bar{t}=t K \in \bar{T}$, define $\bar{x} \cdot \bar{t}=\overline{x t}=p(x t)$. This action is well defined since $K$ is a normal subgroup of $T$ and so $(\bar{X}, \bar{T})$ is a tg since $(X, T)$ is.

Now it follows in a straightforward manner that $p\left(S_{1}\right)=\bar{S}_{1}$ and $p\left(S_{2}\right)=\bar{S}_{2}$ are closed $\overline{1}$-slices in the $\operatorname{tg}(\bar{X}, \bar{T})$ where $\overline{1}$ represents the trivial subgroup of $\bar{T}$. The lemma applies now and we have a closed $\overline{1}$-slice $\bar{S}$ with $\bar{S} \bar{T}=\bar{S}_{1} \bar{T} \cup \bar{S}_{2} \bar{T}$. Let $S=p^{-1}(\bar{S})$ and again it follows that $S$ is a closed $K$-slice in the $\operatorname{tg}(X, T)$ with the property that $S \supset S_{1}$ and $S T=S_{1} T \cup S_{2} T$.

THEOREM 5. Let $X$ be a normal Lindelöf space and $(X, T)$ a tg with $T=K V, a$ semidirect product of $K$ a compact normal Lie subgroup of $T$ and $V$ a vector group. Then $(X, T)$ is a proper $t g$ if and only if $X$ has a global $K$-slice.

Proof. Since $(X, T)$ is a proper tg all isotropy subgroups are compact and so lie in $K$. From results of Palais [4], there is a slice at each point of $X$ and $X / T$ is regular. Consequently, if $S$ is a slice at $x \in X$ there is a closed slice $S_{x}$ at $x$ with $S_{x} \subset S$. By definition of closed slice there is an $S_{x}^{1} \subset S_{x}$ such that $x \in S_{x}^{1}$ and $S_{x}^{1} T$ is open. It follows then since there is a slice at each point of $X$ that $\left\{S_{x}^{1} T\right\}_{x \in X}$ covers $X . X$ is Lindelöf so that a countable subcollection $\left\{S_{x_{i}}^{1} T\right\}_{i=1}^{\infty}$ still covers $X$. Let $S_{x_{i}}$ be the closed slice corresponding to $S_{x_{i}}^{1}$. We now construct a sequence of closed $K$-slices whose union is a global $K$-slice.

Let $S(1)=S_{x_{1}} . S(2)$ is the closed slice obtained from $S(1)$ and $S_{x_{2}}$ by means of Theorem 4, with $S(2) \supset S(1)$. Continuing inductively, $S(n)$ is the closed slice obtained from $S(n-1)$ and $S_{x_{n}}$ with $S(n) \supset S(n-1)$. It is easy to see that for any $n$, $S(n) T \supset \bigcup_{i=1}^{n} S_{x_{i}}^{1} T$. Define $S=\bigcup_{i=1}^{\infty} S(i)$. We claim that $S$ is a global $K$-slice. In fact, it is clear that $S T=X$ and that there is an equivariant map $f: S T \rightarrow T / K$ with 
$f^{-1}(K)=S$. We show that $f$ is continuous. Let $t \in T, s \in S$ with $s t \in S T$. There exists an integer $i$ with $s t \in S(i) T$ such that $S(i) T$ is a neighborhood of st. Let $f(i): S(i) T \rightarrow T / K$ be the continuous equivariant map defining the closed $K$-slice $S(i)$. It is clear that $\left.f\right|_{S(i) T}=f(i)$ and so $f$ is continuous on $S(i) T$, a neighborhood of $s t$. We conclude that $f$ is therefore continuous and the proof of this implication is complete.

For the converse statement, let $S$ be a global $K$-slice. By a result of Palais [4], there exists a neighborhood $U$ of $S$ such that the closure of $T(U, U)$ is compact. Hence $U T=X$ and $(X, T)$ is a proper tg.

\section{REFERENCES}

1. H. Chu, On the existence of slices for transformation groups, Proc. Amer. Math. Soc. 18 (1967), 513-517. MR 35 \#3001.

2. J. Dugundji and H. A. Antosiewicz, Parallelizable flows and Lyapunov's second method, Ann. of Math. (2) 73 (1961), 543-555. MR 23 \#A395.

3. W. H. Gottschalk and G. A. Hedlund, Topological dynamics, Amer. Math. Soc. Colloq. Publ., vol. 36, Amer. Math. Soc., Providence, R. I., 1955. MR 17, 650.

4. R. S. Palais, On the existence of slices for actions of non-compact Lie groups, Ann. of Math. (2) 73 (1961), 295-323. MR 23 \#A3802.

UNIVERSITY OF MARYLAND, College Park, Maryland UNIVERSITY OF MASSACHUSETTS, AMHerst, MassachusetTS 